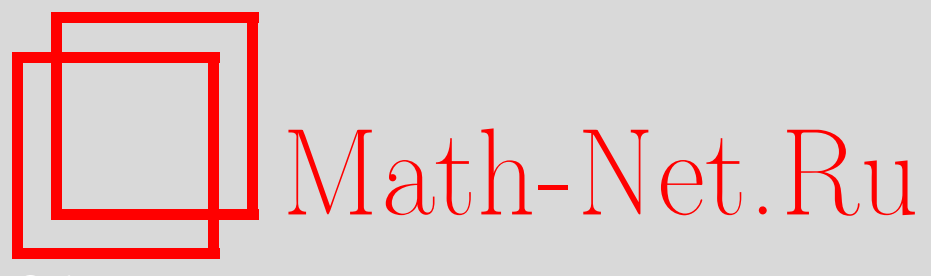

В. Ф. Бабенко, Н. В. Парфинович, О точных значениях наилучших приближений классов дифференцируемых периодических функций сплайнами, Матем. заметки, 2010, том 87, выпуск 5, 669-683

DOI: https://doi.org/10.4213/mzm8716

Использование Общероссийского математического портала Math-Net.Ru подразумевает, что вы прочитали и согласны с пользовательским соглашением http: //www . mathnet.ru/rus/agreement

Параметры загрузки:

IP: 34.229 .108 .108

26 апреля 2023 г., 12:49:13

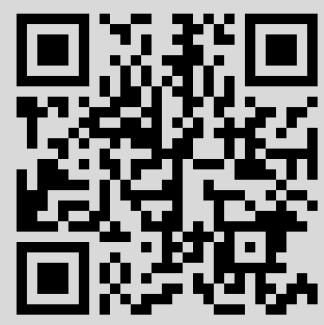


Том 87 выпуск 5 май 2010

УДК 517

\section{О точных значениях наилучших приближений классов дифференцируемых периодических функций сплайнами}

\section{В. Ф. Бабенко, Н. В. Парфинович}

Найдены точные значения наилучших $L_{1}$-приближений классов $W^{r} F, r \in \mathbb{N}$, периодических функций, $r$-я производная которых принадлежит заданному перестановочно-инвариантному множеству $F$, а также классов $W^{r} H^{\omega}$ периодических функций, $r$-я производная которых имеет заданную выпуклую вверх мажоранту $\omega(t)$ модуля непрерывности, подпространствами полиномиальных сплайнов порядка $m \geqslant r+1$ дефекта 1 с узлами в точках $2 k \pi / n$ и $2 k \pi / n+h$, $n \in \mathbb{N}, k \in \mathbb{Z}, h \in(0,2 \pi / n)$. Показано, что эти подпространства являются экстремальными для поперечников по Колмогорову соответствующих функциональных классов.

Библиография: 26 названий.

1. Необходимые обозначения и определения. Пусть $C$ и $L_{p}, 1 \leqslant p \leqslant \infty$, пространства $2 \pi$-периодических функций $f: \mathbb{R} \rightarrow \mathbb{R}$ с соответствующими нормами $\|\cdot\|_{C}$ и $\|\cdot\|_{L_{p}}=\|\cdot\|_{p}, p^{\prime}=p /(p-1)$. Через $L_{p}^{r}, r=1,2, \ldots$, обозначим множество $2 \pi$-периодических функций $f$ таких, что $f^{(r-1)}\left(f^{(0)}:=f\right)$ локально абсолютно непрерывна и $f^{(r)} \in L_{p}$, а через $C^{r}, r=1,2, \ldots$, - пространство $r$ раз непрерывно дифференцируемых (непрерывных при $r=0$ ) $2 \pi$-периодических функций.

Пусть $X$ есть $C$ или $L_{p}, 1 \leqslant p<\infty$. Модулем непрерывности функции $f \in X$ в пространстве $X$ (см. [1; $\S 6.1])$ называется функция

$$
\omega(f, \delta)_{X}:=\sup _{0 \leqslant h \leqslant \delta}\|f(\cdot+h)-f(\cdot)\|_{X}, \quad \delta \geqslant 0 .
$$

Если $X=L_{p}, 1 \leqslant p<\infty$, то вместо $\omega(f, \delta)_{L_{p}}$ будем писать $\omega(f, \delta)_{p}$, а вместо $\omega(f, \delta)_{C}$ будем писать $\omega(f, \delta)_{\infty}$.

Пусть $H$ - подпространство пространства $L_{p}$ и $g \in L_{p^{\prime}}$. Если для всех $h \in H$

$$
\int_{0}^{2 \pi} g(x) h(x) d x=0,
$$

то будем писать $g \perp H$. Если $H$ - подпространство констант, то вместо $g \perp H$ будем использовать обозначение $g \perp 1$.

Пусть множество $F \subset L_{1}$ таково, что $\{f \in F: f \perp 1\} \neq \varnothing$. Для $r=1,2, \ldots$ обозначим через $W^{r} F$ класс функций $f \in L_{1}$, у которых $(r-1)$-я производная $f^{(r-1)}$

(C) В.Ф. БАБенко, Н. В. ПАРФинович, 2010 
$\left(f^{(0)}=f\right)$ локально абсолютно непрерывна и $f^{(r)} \in F$. Отметим, что если $F-$ единичный шар пространства $L_{p}$, то множество $W^{r} F=W_{p}^{r}$ - это стандартный соболевский класс функций, у которых $(r-1)$-я производная локально абсолютно непрерывна и $\left\|f^{(r)}\right\|_{p} \leqslant 1$.

Для неотрицательной функции $f \in L_{1}$ обозначим через $r(f, t)$ неубывающую перестановку (см. [2; с. 130]) сужения функции $f$ на промежуток $[0,2 \pi]$. Если $g-$ произвольная функция из $L_{1}$, то положим (см. [3; с. 99])

$$
\Pi(g, t):=r\left(g_{+}, t\right)-r\left(g_{-}, 2 \pi-t\right)
$$

где $g_{ \pm}:=\max \{ \pm g(t), 0\}$.

Множество $F \in L_{1}$ назовем П-инвариантным, если из того, что $f \in F$ и $\Pi(g)=$ $\Pi(f)$, следует, что $g \in F$.

Отметим, что П-инвариантным является, например, единичный шар в любом вложенном в $L_{1}$ симметричном пространстве $2 \pi$-периодических функций, в частности, в пространствах $L_{p}, 1 \leqslant p \leqslant \infty$, Орлича [4], Лоренца [5], [6] и Марцинкевича [5], [6]. Другие примеры П-инвариантных множеств можно найти, например, в [7] и [8].

Пусть $\omega(t)$ - произвольный фиксированный модуль непрерывности, т.е. непрерывная на $[0,+\infty)$, неубывающая, полуаддитивная функция, в нуле равная нулю. Класс функций $f \in C^{r}$ таких, что $\omega(f, t)_{\infty} \leqslant \omega(t), t \geqslant 0$, будем обозначать через $W^{r} H^{\omega}$.

Наилучшим приближением функции $f \in L_{p}$ множеством $H \subset L_{p}$ в метрике $L_{p}$ называется величина

$$
E(f, H)_{p}=\inf _{h \in H}\|f-h\|_{p}
$$

Наилучшее приближение класса $M \subset L_{p}$ множеством $H$ из $L_{p}$ в метрике $L_{p}$ - это величина

$$
E(M, H)_{p}=\sup _{f \in M} E(f, H)_{p}
$$

Для $n \in \mathbb{N}$ величина

$$
d_{n}\left(M, L_{p}\right)=\inf _{H_{n}} E\left(M, H_{n}\right)_{p}
$$

где $\inf _{H_{n}}$ берется по всем подпространствам $H_{n}$ пространства $L_{p}$, размерность которых не превосходит $n$, называется $n$-поперечником Колмогорова класса $M$ в пространстве $L_{p}$. Подпространства $H_{n}$, которые реализуют точную нижнюю грань в правой части (1.1), называются экстремальными.

Для каждого натурального $n$ и $m=0,1, \ldots$ через $T_{2 n-1}$ обозначим пространство тригонометрических полиномов порядка не выше $n-1$, через $S_{2 n, m}^{1}$ - пространство $2 \pi$-периодических полиномиальных сплайнов порядка $m$ дефекта 1 с узлами в точках $j \pi / n, j \in \mathbb{Z}$, а через $S_{2 n, m}^{2}, n, m \in \mathbb{N},-$ пространство $2 \pi$-периодических полиномиальных сплайнов порядка $m$ дефекта 2 с узлами в точках $t_{j}=2 j \pi / n$, $j \in \mathbb{Z}$.

Пусть $\varphi_{\lambda, m}(t), m \in \mathbb{N}, \lambda>0,-m$-й $2 \pi / \lambda$-периодический интеграл с нулевым средним значением на периоде от функции $\varphi_{\lambda, 0}(t)=\operatorname{sign} \sin \lambda t$. 
При фиксированных $\omega(\cdot)$ и $n \in \mathbb{N}$ положим

$$
f_{n, 0}(\omega, t)= \begin{cases}-\frac{1}{2} \omega\left(\frac{\pi}{n}-2 t\right), & 0 \leqslant t \leqslant \frac{\pi}{2 n}, \\ \frac{1}{2} \omega\left(2 t-\frac{\pi}{n}\right), & \frac{\pi}{2 n} \leqslant t \leqslant \frac{\pi}{n} .\end{cases}
$$

Через $f_{n, r}(\omega, t), r=1,2, \ldots$, обозначим $r$-й $2 \pi / n$-периодический интеграл от $f_{n, 0}(\omega, t)$ с нулевым средним значением на периоде.

2. Некоторые известные результаты. Пусть $n, r \in \mathbb{N}, m \in \mathbb{N} \cup\{0\}, 1 \leqslant p \leqslant \infty$, $H$ есть $T_{2 n-1}, S_{2 n, m}^{1}, m \geqslant r-1$, или $S_{2 n, m}^{2}, m \geqslant r$. Тогда

$$
\begin{gathered}
E\left(W_{p}^{r}, H\right)_{1}=\left\|\varphi_{n, r}\right\|_{p^{\prime}}, \\
d_{2 n-1}\left(W_{p}^{r}, L_{1}\right)=d_{2 n}\left(W_{p}^{r}, L_{1}\right)=\left\|\varphi_{n, r}\right\|_{p^{\prime}}
\end{gathered}
$$

и, следовательно, подпространства $T_{2 n-1}$ являются экстремальными для поперечников $d_{2 n-1}\left(W_{p}^{r}, L_{1}\right)$ и $d_{2 n}\left(W_{p}^{r}, L_{1}\right)$, а пространства $S_{2 n, m}^{1}, m \geqslant r-1$, и $S_{2 n, m}^{2}, m \geqslant r,-$ для поперечников $d_{2 n}\left(W_{p}^{r}, L_{1}\right)$.

Равенство (2.1) при $p=1$ и $H=T_{2 n-1}$ получено Никольским [9]; для $p>1$ его установил Тайков [10]; при $p=\infty$ независимо и другим методом этот результат получила Туровец [11]. Для $H=S_{2 n, m}^{1}, m \geqslant r-1$, равенство (2.2) установлено Лигуном [12], а для $H=S_{2 n, m}^{2}, m \geqslant r,-$ Бабенко и Парфинович [8]. Оценку снизу для нечетных поперечников при $p=1$ получили независимо Маковоз [13] и Субботин [14], [15], а при $p=\infty$ Маковоз [16]. Оценка снизу для четных поперечников при $p=1, \infty$ принадлежит Рубану (см., например, [2; гл. 10]). Для $1<p<\infty$ соотношение (2.2) независимо и разными методами получили Лигун [17], Маковоз [18], Пинкус [19].

Для произвольного П-инвариантного множества $F$ и любых $n, r=1,2, \ldots$ имеют место следующие равенства:

$$
E\left(W^{r} F, H\right)_{1}=\sup _{\substack{f \in F \\ f \perp 1}} \int_{0}^{2 \pi} \Pi(f, t) \Pi\left(\varphi_{n, r}, t\right) d t
$$

где, как и выше, $H$ есть $T_{2 n-1}, S_{2 n, m}^{1}$ или $S_{2 n, m}^{2}, m \geqslant r$. Кроме того,

$$
d_{2 n-1}\left(W^{r} F, L_{1}\right)=d_{2 n}\left(W^{r} F, L_{1}\right)=\sup _{\substack{f \in F \\ f \perp 1}} \int_{0}^{2 \pi} \Pi(f, t) \Pi\left(\varphi_{n, r}, t\right) d t,
$$

откуда следует, что подпространства $T_{2 n-1}$ являются экстремальными для поперечников $d_{2 n-1}\left(W^{r} F, L_{1}\right)$ и $d_{2 n}\left(W^{r} F, L_{1}\right)$, а подпространства $S_{2 n, m}^{1}$ и $S_{2 n, m}^{2}$ при всех $m \geqslant r$ - для поперечников $d_{2 n}\left(W^{r} F, L_{1}\right)$.

Для $H=T_{2 n-1}$ и $H=S_{2 n, m}^{1}$ соотношение (2.3) доказано в [7], а для $H=S_{2 n, m}^{2}-$ в [8]. Соотношение (2.4) получено в [7].

Корнейчук [20]-[22] (см. также [1; теоремы 7.2.2, 7.2.5]) доказал, что для любого выпуклого вверх модуля непрерывности $\omega(t)$, при всех $n=1,2, \ldots, r=0,1, \ldots$, $m \geqslant r$ имеет место соотношение

$$
E\left(W^{r} H^{\omega}, T_{2 n-1}\right)_{1}=E\left(W^{r} H^{\omega}, S_{2 n, m}^{1}\right)_{1}=\left\|f_{n, r}(\omega, \cdot)\right\|_{1} .
$$


Известно также, что

$$
d_{2 n-1}\left(W^{r} H^{\omega}, L_{1}\right)=d_{2 n}\left(W^{r} H^{\omega}, L_{1}\right)=\left\|f_{n, r}(\omega, \cdot)\right\|_{1},
$$

причем поперечники $d_{2 n-1}\left(W^{r} H^{\omega}, L_{1}\right)$ и $d_{2 n}\left(W^{r} H^{\omega}, L_{1}\right)$ реализуют подпространство $T_{2 n-1}$, а поперечник $d_{2 n}\left(W^{r} H^{\omega}, L_{1}\right)$ - подпространство $S_{2 n, m}^{1}, m \geqslant r$. Оценки снизу для нечетных поперечников получены Моторным и Рубаном [23], а для четных Рубаном [24].

В работе [8] найдены точные значения наилучших $L_{1}$-приближений классов $W^{r} H^{\omega}$ сплайнами из $S_{2 n, m}^{2}$ при $m \geqslant r+1$, а именно, доказано, что для любых $n, r=1,2, \ldots$ и любого выпуклого вверх модуля непрерывности $\omega(t)$ при всех $m \geqslant r+1$ имеет место равенство

$$
E\left(W^{r} H^{\omega}, S_{2 n, m}^{2}\right)_{1}=\left\|f_{n, r}(\omega, \cdot)\right\|_{1},
$$

так что и подпространства $S_{2 n, m}^{2}, m \geqslant r+1$, являются экстремальными подпространствами для поперечников $d_{2 n}\left(W^{r} H^{\omega}, L_{1}\right)$.

Пусть $r=0,1,2, \ldots, 1 \leqslant p, q \leqslant \infty, H$ - подпространство пространства $L_{p}, \delta>0$. Неравенствами типа Джексона называются неравенства вида

$$
E(f, H)_{p} \leqslant K \omega\left(f^{(r)}, \delta\right)_{q},
$$

справедливые для любой достаточно гладкой функции $f$ с константой $K$, не зависящей от $f$.

Много работ было посвящено отысканию неравенств типа Джексона с неулучшаемыми константами. Обзор известных результатов и соответствующие ссылки можно найти, например, в [1] и [25]. Нам понадобится следующая теорема, доказанная Лигуном (см. [25; теорема 14]).

Теорема А. Пусть $r=1,2, \ldots, H$ - произвольное подпространство пространства $L_{1}$, содержащее константы, и

$$
A_{r, 1}=\left(\left\|\varphi_{1, r}\right\|_{\infty}^{-1} E\left(W_{1}^{r}, H\right)_{1}\right)^{1 / r} .
$$

Тогда для любой функиии $f \in L_{1}^{r}$ при $r=1,3,5, \ldots u \delta \geqslant \pi A_{r+1,1}$

$$
E(f, H)_{1} \leqslant \frac{1}{2} A_{r+1,1}^{r}\left\|\varphi_{1, r}\right\|_{\infty} \cdot \omega\left(f^{(r)}, \delta\right)_{1} .
$$

Для любой функиии $f \in C^{r}, r=1,2,3, \ldots$, при $\delta \geqslant 2 \pi A_{r+2,1}$

$$
E(f, H)_{1} \leqslant 2 A_{r+2,1}^{r}\left\|\varphi_{1, r+1}\right\|_{\infty} \cdot \omega\left(f^{(r)}, \delta\right)_{\infty} .
$$

Из теоремы А и приведенных выше результатов о наилучших приближениях классов $W_{p}^{r}, p=1, \infty$, тригонометрическими полиномами и сплайнами следует ряд точных неравенств типа Джексона (см. [25; следствия 1, 2 из теоремы 14]). Некоторые из этих точных неравенств были доказаны ранее и другими методами (см., например, [1; гл. 6]).

Приведем точные неравенства типа Джексона для наилучших $L_{1}$-приближений сплайнами. Пусть $n, m, r \in \mathbb{N}, H$ есть $S_{2 n, m}^{1}, m \geqslant r$, или $S_{2 n, m}^{2}, m \geqslant r+1$. Тогда для любой функции $f \in L_{1}^{r}, r=1,3,5, \ldots$, при любом $\delta \geqslant \pi n^{-1}$ выполняется точное неравенство

$$
E(f, H)_{1} \leqslant \frac{\left\|\varphi_{1, r}\right\|_{\infty}}{2 n^{r}} \omega\left(f^{(r)}, \delta\right)_{1} .
$$


Если $H$ есть $S_{2 n, m}^{1}, m \geqslant r+1$, или $S_{2 n, m}^{2}, m \geqslant r+2$, то для любой функции $f \in C^{r}$, $r=1,2, \ldots$, при любом $\delta \geqslant 2 \pi n^{-1}$ выполняется точное неравенство

$$
E(f, H)_{1} \leqslant \frac{2\left\|\varphi_{1, r+1}\right\|_{\infty}}{n^{r}} \omega\left(f^{(r)}, \delta\right)_{\infty} .
$$

До сих пор речь шла о вычислении точных констант в неравенствах типа Джексона при приближении фиксированными подпространствами. Рассмотрим задачу минимизации этих констант по всем подпространствам фиксированной размерности. Пусть $X_{q}^{r}$ есть $L_{q}^{r}$, если $q<\infty$, и $X_{q}^{r}$ есть $C^{r}$, если $q=\infty$. Положим для $H \subset L_{p}$

$$
\kappa_{r}(H, \delta)_{p, q}=\sup \left\{\frac{E(f, H)_{p}}{\omega\left(f^{(r)}, \delta\right)_{q}}: f \in X_{q}^{r}, f \neq \text { const }\right\},
$$

т.е. $\kappa_{r}(H, \delta)_{p, q}$ - наименьшая константа $\kappa$ в неравенстве

$$
E(f, H)_{p} \leqslant \kappa \omega\left(f^{(r)}, \delta\right)_{q} .
$$

Рассмотрим задачу вычисления величин

$$
\kappa_{n, r}(\delta)_{p, q}=\inf \left\{\kappa_{r}(H, \delta)_{p, q}: H \subset L_{q}, \operatorname{dim} H \leqslant n\right\} .
$$

Подпространства, реализующие inf в правой части (2.12), называются экстремальными подпространствами в задаче минимизации констант Джексона.

Отметим, что при всех $n, r \in \mathbb{N}, 1 \leqslant p, q \leqslant \infty$

$$
\kappa_{n, r}(\delta)_{p, q} \geqslant \frac{1}{2} d_{n}\left(\widetilde{W}_{q}^{r}, L_{p}\right)=\frac{1}{2} d_{n}\left(W_{q}^{r}, L_{p}\right),
$$

где $\widetilde{W}_{q}^{r}=W_{p}^{r}$, если $q<\infty$, и $\widetilde{W}_{\infty}^{r}=\left\{f \in W_{\infty}^{r}: f^{(r)} \in C\right\}$. Неравенство

$$
\kappa_{n, r}(\delta)_{p, q} \geqslant \frac{1}{2} d_{n}\left(\widetilde{W}_{q}^{r}, L_{p}\right)
$$

при $q<\infty$ отмечено Лигуном (см. [25; неравенство (101)]), неравенство

$$
\kappa_{n, r}(\delta)_{p, \infty} \geqslant \frac{1}{2} d_{n}\left(\widetilde{W}_{\infty}^{r}, L_{p}\right)
$$

устанавливается аналогично. Равенство

$$
d_{n}\left(\widetilde{W}_{q}^{r}, L_{p}\right)=d_{n}\left(W_{q}^{r}, L_{p}\right), \quad 1 \leqslant q \leqslant \infty
$$

очевидно.

Из сопоставления неравенств $(2.10)$ и (2.11) с (2.13) вытекает, что при $r=1,3,5, \ldots$ и $\delta \geqslant \pi n^{-1}$

$$
\kappa_{2 n, r}(\delta)_{1,1}=\kappa_{r}\left(S_{2 n, m}^{1}, \delta\right)_{1,1}=\kappa_{r}\left(S_{2 n, m}^{2}, \delta\right)_{1,1}=\frac{\left\|\varphi_{1, r}\right\|_{\infty}}{2 n^{r}},
$$

а при $r=1,2, \ldots$ и $\delta \geqslant 2 \pi n^{-1}$

$$
\kappa_{2 n, r}(\delta)_{1, \infty}=\kappa_{r}\left(S_{2 n, m}^{1}, \delta\right)_{1, \infty}=\kappa_{r}\left(S_{2 n, m}^{2}, \delta\right)_{1, \infty}=\frac{2\left\|\varphi_{1, r+1}\right\|_{\infty}}{n^{r}} .
$$


Следовательно, экстремальными в задаче вычисления величин $\kappa_{2 n, r}(\delta)_{1,1}$ при $r=$ $1,3,5, \ldots, \delta \geqslant \pi n^{-1}$ и $\kappa_{2 n, r}(\delta)_{1, \infty}$ при $r=1,2, \ldots, \delta \geqslant 2 \pi n^{-1}$ являются подпространства $S_{2 n, m}^{1}$ и $S_{2 n, m}^{2}, m \geqslant r$.

В данной статье мы найдем наилучшее приближение классов $W^{r} F$ и $W^{r} H^{\omega}$ в пространстве $L_{1}$ сплайнами порядка $m$ минимального дефекта с узлами в точках $2 \pi i / n$ и $2 \pi i / n+h, 0<h<2 \pi / n, h \neq \pi / n, i \in \mathbb{Z}$, и для наилучших приближений функции $f \in L_{p}^{r}, p=1, \infty$, такими сплайнами получим точные неравенства типа Джексона. Из этих результатов будет следовать, что подпространства таких сплайнов являются экстремальными подпространствами в задачах о $n$-поперечниках и экстремальными подпространствами в задаче (2.12).

3. Аппроксимация сплайнами $S_{2 n, m}^{1}(h)$ и неравенства типа Джексона. Формулировка результатов. Пусть $n, m \in \mathbb{N}, h \in(0,2 \pi / n)$. Через $S_{2 n, m}^{1}(h)$ будем обозначать пространство сплайнов порядка $m$ дефекта 1 с узлами в точках

$$
t_{j}=\frac{2 \pi[j / 2]}{n}+\left(1-(-1)^{j}\right) \frac{h}{2}, \quad j \in \mathbb{Z},
$$

где [·] - целая часть числа. Отметим, что размерность этого пространства при любом $m$ равна $2 n$ (см., например, [1; следствие 2.3.7]) и $S_{2 n, m}^{1}(\pi / n)=S_{2 n, m}^{1}$.

Нами доказаны следующие теоремы.

Tеорема 1. Пусть $n, m, r \in \mathbb{N}, m \geqslant r+1,0<h<2 \pi / n, 1 \leqslant p \leqslant \infty, F-$ произвольное П-инвариантное множество $2 \pi$-периодических функций. Тогда

$$
E\left(W^{r} F, S_{2 n, m}^{1}(h)\right)_{1}=\sup _{\substack{f \in F \\ f \perp 1}} \int_{0}^{2 \pi} \Pi(f, t) \Pi\left(\varphi_{n, r}, t\right) d t .
$$

В частности,

$$
E\left(W_{p}^{r}, S_{2 n, m}^{1}(h)\right)_{1}=\left\|\varphi_{n, r}\right\|_{p^{\prime}} .
$$

При $p=1$ соотношение (3.2) верно и при $m=r$.

ТеОрема 2. Пусть $n, m, r \in \mathbb{N}, m \geqslant r+1,0<h<2 \pi / n, \omega(t)$ - выпуклый вверх модуль непрерывности. Тогда

$$
E\left(W^{r} H^{\omega}, S_{2 n, m}^{1}(h)\right)_{1}=\left\|f_{n, r}(\omega, \cdot)\right\|_{1} .
$$

Результаты теорем 1 и 2 вместе с соотношениями (2.4) и (2.6) показывают, что $S_{2 n, m}^{1}(h)$ наряду с $T_{2 n-1}, S_{2 n, m}^{1}$ и $S_{2 n, m}^{2}$ являются экстремальными подпространствами для поперечников $d_{2 n}\left(W^{r} F, L_{1}\right)$ и $d_{2 n}\left(W^{r} H^{\omega}, L_{1}\right)$ (при $\left.m \geqslant r+1\right)$.

Теорема 3. Пусть $n, m, r \in \mathbb{N}$. Для любой функиии $f \in L_{1}^{r}$ при $r=1,3,5, \ldots$, $m \geqslant r+1$ и $\delta \geqslant \pi n^{-1}$ выполняется точное неравенство

$$
E\left(f, S_{2 n, m}^{1}(h)\right)_{1} \leqslant \frac{\left\|\varphi_{1, r}\right\|_{\infty}}{2 n^{r}} \omega\left(f^{(r)}, \delta\right)_{1} .
$$

Для любой функции $f \in C^{r}$ при $r=1,2, \ldots, m \geqslant r+2 u \delta \geqslant 2 \pi n^{-1}$ выполняется точное неравенство

$$
E\left(f, S_{2 n, m}^{1}(h)\right)_{1} \leqslant \frac{2\left\|\varphi_{1, r+1}\right\|_{\infty}}{n^{r}} \omega\left(f^{(r)}, \delta\right)_{\infty} .
$$


При доказательстве теорем 1 и 2 нами существенно будет использована следующая теорема, которая представляет и самостоятельный интерес.

Tеорема 4. Пусть $n, l \in \mathbb{N}, \widetilde{\varphi}(t)=\varphi_{n, l}(t+\mu)+\nu$, где $\mu$ и выбраны так, чтобъ $\widetilde{\varphi}\left(t_{j}\right)=0, j \in \mathbb{Z}$. Тогда для любой функиии $g \in W_{\infty}^{l}$ такой, что

$$
g\left(t_{1}\right)=g\left(t_{2}\right)=\cdots=g\left(t_{2 n}\right)=0,
$$

имеют место следующие утверждения:

а) для всех $t \in \mathbb{R}$ выполнено $|g(t)| \leqslant|\widetilde{\varphi}(t)|$, причем, если $g(t)$ отлична от $\pm \widetilde{\varphi}(t)$, то знак равенства имеет место только в точках $t_{j}, j=1,2, \ldots, 2 n$;

b) $\left\|g^{(k)}\right\|_{\infty} \leqslant\left\|\varphi_{n, l-k}\right\|_{\infty}, k=1,2, \ldots, l-1$.

4. Доказательства. Сначала мы докажем теорему 4, а затем теоремы 1 и 2. Теорема 3 будет получена сопоставлением теоремы А и соотношения (3.2) при $p=1$.

ДоКАЗАТЕЛЬСТво теоремЫ 4. Функции $\widetilde{\varphi}(t)$ и $g(t)$ имеют нули в точках $t_{j}$, $j=1, \ldots, 2 n$. Для доказательства утверждения а) предположим, что найдется $t_{*}$ такое, что $\left|g\left(t_{*}\right)\right|>\left|\widetilde{\varphi}\left(t_{*}\right)\right|$. Считая для определенности, что $\widetilde{\varphi}\left(t_{*}\right)>0$, при подходящем $\lambda, 0<|\lambda|<1$, получим $\lambda g\left(t_{*}\right)=\widetilde{\varphi}\left(t_{*}\right)$. Пусть $\delta(t)=\widetilde{\varphi}(t)-\lambda g(t)$. Так как $\left\|\lambda g^{(l)}\right\|_{\infty}<\left\|\widetilde{\varphi}^{(l)}\right\|_{\infty}$, то $\delta$ имеет только изолированные нули. Количество этих нулей не менее $2 n+1$ и, следовательно, с учетом периодичности $\delta$ имеет на периоде не менее $2 n+2$ нулей с учетом кратности, причем не менее $2 n+1$ из них различны; следовательно, $\delta^{\prime}, \delta^{\prime \prime}, \ldots, \delta^{(l)}$ имеют на периоде не менее $2 n+2$ перемен знака. Однако, $\delta^{(l)}(t)=\varphi_{n, 0}(t)-\lambda g^{(l)}(t)$ имеет на периоде ровно $2 n$ перемен знака, и мы получили противоречие, которое доказывает утверждение а).

Докажем утверждение b). Для определенности будем считать, что $\widetilde{\varphi}(t)<0$ для $t \in\left(t_{2 i}, t_{2 i+1}\right), i \in \mathbb{Z}$. Пусть $t_{\max } \in(0,2 \pi)$ таково, что $\left|g^{\prime}\left(t_{\max }\right)\right|=\left\|g^{\prime}\right\|_{\infty}$, и $i \in \mathbb{Z}$ выбрано из условия $t_{\max } \in\left(t_{2 i}, t_{2 i+2}\right)$. Предположив, что $\left|g^{\prime}\left(t_{\max }\right)\right|>\left\|\widetilde{\varphi}^{\prime}\right\|_{\infty}$, мы сможем указать такое $0<|\lambda|<1$, что

$$
\lambda g^{\prime}\left(t_{\max }\right)=\left\|\widetilde{\varphi}^{\prime}\right\|_{\infty}=\widetilde{\varphi}^{\prime}\left(t_{\max }^{1}\right)=\left|\widetilde{\varphi}^{\prime}\left(t_{\min }^{1}\right)\right|
$$

где $t_{\max }^{1}<t_{\min }^{1}$ - точки из промежутка $\left[t_{2 i}, t_{2 i+2}\right]$, в которых функция $\widetilde{\varphi}^{\prime}(t)$ принимает соответственно максимальное и минимальное значения. Ясно, что точки $t_{\max }^{1}$, $t_{\text {min }}^{1}$ принадлежат промежутку $\left(t_{2 i+1}, t_{2 i+2}\right)$.

Покажем, что $t_{\max } \in\left(t_{\max }^{1}, t_{\min }^{1}\right)$. Предположим, что $t_{\max } \leqslant t_{\max }^{1}$. Случай $t_{\max } \geqslant$ $t_{\min }^{1}$ рассматривается аналогично.

Отметим, что для функций $\lambda g^{\prime}(t)$ и $\widetilde{\varphi}^{\prime}(t)$ выполнены условия теоремы сравнения производных [1; теорема 3.3.2], причем $\lambda g^{\prime}(t) \neq \widetilde{\varphi}^{\prime}(t)$ почти всюду. Обозначим через $t^{1}$ ближайший слева, а через $t^{2}$ ближайший справа к точке $t_{\max }$ нуль функции $\lambda g^{\prime}(t)$; тогда с помощью предложения 3.3 .3 из [1; теорема 3.3.2] нетрудно убедиться в том, что

$$
t_{\max }-t^{1}>\frac{\pi}{2 n}=t_{\max }^{1}-\frac{t_{2 i}+t_{2 i+1}}{2}
$$

так что

$$
t^{1}<t_{\max }-t_{\max }^{1}+\frac{t_{2 i}+t_{2 i+1}}{2} \leqslant \frac{t_{2 i}+t_{2 i+1}}{2},
$$


и, кроме того, для любого $t \in\left(t_{2 i}, t_{2 i+1}\right)$ будет $\lambda g^{\prime}(t)>\widetilde{\varphi}^{\prime}(t)$ Тогда

$$
\begin{gathered}
\int_{t^{1}}^{t_{2 i+1}} \lambda g^{\prime}(t) d t>\int_{\left(t_{2 i}+t_{2 i+1}\right) / 2}^{t_{2 i+1}} \widetilde{\varphi}^{\prime}(t) d t \\
\left|\int_{t_{2 i}}^{t^{1}} \lambda g^{\prime}(t) d t\right|<\left|\int_{t_{2 i}}^{\left(t_{2 i}+t_{2 i+1}\right) / 2} \widetilde{\varphi}^{\prime}(t) d t\right|=\int_{\left(t_{2 i}+t_{2 i+1}\right) / 2}^{t_{2 i+1}} \widetilde{\varphi}^{\prime}(t) d t,
\end{gathered}
$$

что противоречит тому факту, что $\int_{t_{2 i}}^{t_{2 i+1}} \lambda g^{\prime}(t) d t=0$.

Покажем, что функция $\lambda g^{\prime}(t)$ имеет нуль на промежутке $\left(t_{2 i+1}, t_{\max }\right)$. Аналогично предыдущему устанавливаем, что

$$
t_{\max }-t_{2 i+1}>t_{\max }^{1}-t_{2 i+1}, \quad t^{2}-t_{\max }>\frac{t_{2 i+1}+t_{2 i+2}}{2}-t_{\max }^{1}
$$

и что

$$
\int_{t_{2 i+1}}^{t_{\max }} \lambda g^{\prime}(t) d t>\int_{t_{2 i+1}}^{t_{\max }^{1}} \widetilde{\varphi}^{\prime}(t) d t, \quad \int_{t_{\max }}^{t^{2}} \lambda g^{\prime}(t) d t>\int_{t_{\max }^{1}}^{\left(t_{2 i+1}+t_{2 i+2}\right) / 2} \widetilde{\varphi}^{\prime}(t) d t .
$$

Учитывая еще тот факт, что $g\left(t_{2 i+1}\right)=\widetilde{\varphi}^{\prime}\left(t_{2 i+1}\right)=0$, получаем

$$
\begin{aligned}
\lambda g\left(t^{2}\right) & =\int_{t_{2 i+1}}^{t^{2}} \lambda g^{\prime}(t) d t=\int_{t_{2 i+1}}^{t_{\max }} \lambda g^{\prime}(t) d t+\int_{t_{\max }}^{t^{2}} \lambda g^{\prime}(t) d t \\
& >\int_{t_{2 i+1}}^{t_{\max }^{1}} \widetilde{\varphi}^{\prime}(t) d t+\int_{t_{\max }^{1}}^{\left(t_{2 i+1}+t_{2 i+2}\right) / 2} \widetilde{\varphi}^{\prime}(t) d t \\
& =\int_{t_{2 i+1}}^{\left(t_{2 i+1}+t_{2 i+2}\right) / 2} \widetilde{\varphi}^{\prime}(t) d t=\widetilde{\varphi}\left(\frac{t_{2 i+1}+t_{2 i+2}}{2}\right)=\|\widetilde{\varphi}\|_{\infty} ;
\end{aligned}
$$

следовательно, $\lambda g\left(t^{2}\right)>\|\widetilde{\varphi}\|_{\infty}$, и мы получили противоречие. Таким образом, $t^{1} \in$ $\left(t_{2 i+1}, t_{\max }\right), t_{\max }-t^{1}>\pi / 2 n$ и $t^{2}-t_{\max }>\pi / 2 n$, так что

$$
\begin{gathered}
t^{2}-t^{1}>\frac{\pi}{n} \\
\int_{t^{1}}^{t^{2}} \lambda g^{\prime}(t) d t>\int_{\left(t_{2 i}+t_{2 i+1}\right) / 2}^{\left(t_{2 i+1}+t_{2 i+2}\right) / 2} \widetilde{\varphi}^{\prime}(t) d t
\end{gathered}
$$

(здесь снова нужно воспользоваться предложением 3.3 .3 из [1]). Но тогда с учетом условий $\int_{t_{2 i+1}}^{t_{2 i+3}} \lambda g^{\prime}(t) d t=0$ и $\int_{t_{2 i+1}}^{t_{2 i+3}} \widetilde{\varphi}^{\prime}(t) d t=0$, должно также выполняться неравенство

$$
\left|\int_{\left(t_{2 i+1}, t_{2 i+3}\right) \backslash\left(t^{1}, t^{2}\right)} \lambda g^{\prime}(t) d t\right|>\int_{\left(t_{2 i}+t_{2 i+1}\right) / 2}^{\left(t_{2 i+1}+t_{2 i+2}\right) / 2} \widetilde{\varphi}^{\prime}(t) d t .
$$

Пусть $\left(a_{k}, b_{k}\right)$ - составляющие интервалы множества

$$
\left\{t \in\left(t_{2 i+1}, t_{2 i+3}\right): g^{\prime}(t)<0\right\} .
$$

Тогда в силу (4.2)

$$
\sum_{k}\left(a_{k}, b_{k}\right) \leqslant t_{2 i+3}-t_{2 i+1}-\left(t^{2}-t^{1}\right)<\frac{\pi}{n}=\left.\operatorname{mes} \operatorname{supp} \widetilde{\varphi}_{-}^{\prime}\right|_{(2 i+1,2 i+3)}(t) .
$$


Пусть также для каждого $k$ число $\gamma_{k} \geqslant 0$ таково, что

$$
\left.\left(a_{k}+\gamma_{k}, b_{k}+\gamma_{k}\right) \subset \operatorname{supp} \widetilde{\varphi}_{-}^{\prime}\right|_{(2 i+1,2 i+3)}(t) .
$$

Используя предложение 3.3 .3 из [1] убеждаемся в том, что

$$
\left|\int_{a_{k}+\gamma_{k}}^{b_{k}+\gamma_{k}} g^{\prime}\left(x-\gamma_{k}\right) d x\right| \leqslant\left|\int_{a_{k}+\gamma_{k}}^{b_{k}+\gamma_{k}} \widetilde{\varphi}^{\prime}(t) d t\right| .
$$

Очевидно, что числа $\gamma_{k}$ можно выбрать так, чтобы интервалы $\left(a_{k}+\gamma_{k}, b_{k}+\gamma_{k}\right)$ попарно не пересекались. Тогда будем иметь

$$
\begin{aligned}
\left|\int_{\left(t_{2 i+1}, t_{2 i+3}\right) \backslash\left(t^{1}, t^{2}\right)} \lambda g^{\prime}(t) d t\right| & =\left|\sum_{k} \int_{a_{k}}^{b_{k}} \lambda g^{\prime}(t) d t\right|=\left|\sum_{k} \int_{a_{k}+\gamma_{k}}^{b_{k}+\gamma_{k}} \lambda g^{\prime}\left(t-\gamma_{k}\right) d t\right| \\
& =\sum_{k}\left|\int_{a_{k}+\gamma_{k}}^{b_{k}+\gamma_{k}} \lambda g^{\prime}\left(t-\gamma_{k}\right) d t\right| \leqslant \sum_{k}\left|\int_{a_{k}+\gamma_{k}}^{b_{k}+\gamma_{k}} \widetilde{\varphi}_{-}^{\prime}(t) d t\right| \\
& =\left|\sum_{k} \int_{a_{k}+\gamma_{k}}^{b_{k}+\gamma_{k}} \widetilde{\varphi}_{-}^{\prime}(t) d t\right| \leqslant-\int_{\left(t_{2 i+1}+t_{2 i+2}\right) / 2}^{\left(t_{2 i+2}+t_{2 i+3}\right) / 2} \widetilde{\varphi}^{\prime}(t) d t \\
& =\int_{\left(t_{2 i}+t_{2 i+1}\right) / 2}^{\left(t_{2 i+1}+t_{2 i+2}\right) / 2} \widetilde{\varphi}^{\prime}(t) d t .
\end{aligned}
$$

Таким образом,

$$
\left|\int_{\left(t_{2 i+1}, t_{2 i+3}\right) \backslash\left(t^{1}, t^{2}\right)} \lambda g^{\prime}(t) d t\right| \leqslant \int_{\left(t_{2 i}+t_{2 i+1}\right) / 2}^{\left(t_{2 i+1}+t_{2 i+2}\right) / 2} \widetilde{\varphi}^{\prime}(t) d t,
$$

что противоречит (4.3).

Таким образом, нами доказана справедливость неравенства

$$
\left\|g^{\prime}\right\|_{\infty} \leqslant\left\|\varphi_{n, l-1}\right\|_{\infty}
$$

откуда, используя теорему сравнения [1; теорема 3.3 .2$]$, получим неравенства

$$
\left\|g^{(k)}\right\|_{\infty} \leqslant\left\|\varphi_{n, l-k}\right\|_{\infty}, \quad k=1,2, \ldots, l-1
$$

Теорема доказана.

ДОКАЗАТЕЛЬСТвО ТЕОРЕмЫ 1. Используя теорему двойственности Никольского [9] для наилучшего $L_{1}$-приближения подпространством (см. также [1; теорема 1.4.1]), получим

$$
E:=E\left(W^{r} F, S_{2 n, m}^{1}(h)\right)_{1}=\sup _{f \in W^{r} F} \sup _{\substack{\|g\|_{\infty} \leqslant 1 \\ g \perp S_{2 n, m}^{1}(h)}} \int_{0}^{2 \pi} f(t) g(t) d t .
$$

После $r$-кратного интегрирования по частям с учетом смысла ортогональности функций подпространству $S_{2 n, m}^{1}(h)$ (см. $\left.[1 ; \S 5.4]\right)$ можем написать

$$
E=\sup _{\substack{f \in F \\ f \perp 1}} \sup _{\substack{g \in W_{\infty}^{r} \\ g^{(r)} \perp S_{2 n, m}^{1}(h)}} \int_{0}^{2 \pi} f(t) g(t) d t=\sup _{\substack{g \in W_{\infty}^{m+1} \\ g\left(t_{1}\right)=\cdots=g\left(t_{2 n}\right)=0}} \sup _{\substack{f \in F \\ f \perp 1}} \int_{0}^{2 \pi} f(t) g^{(m-r+1)}(t) d t .
$$


Учитывая предложение 1.3.4 из [26], окончательно получаем

$$
\begin{aligned}
E= & \sup _{\substack{f \in F \\
f \perp 1}} \sup _{\substack{g \in W_{\infty}^{r}, g^{(r)} \perp S_{2 n, m}^{1}(h)}} \int_{0}^{2 \pi} f(t) g(t) d t \\
= & \sup _{\substack{g \in W_{\infty}^{m+1} \\
g\left(t_{1}\right)=\cdots=g\left(t_{2 n}\right)=0}} \sup _{\substack{f \in F \\
f \perp 1}} \int_{0}^{2 \pi} \Pi(f, t) \Pi\left(g^{(m-r+1)}, t\right) d t .
\end{aligned}
$$

В случае, когда $F$ есть единичный шар пространства $L_{1}$, из (4.6) получаем

$$
E\left(W_{1}^{r}, S_{2 n, m}^{1}(h)\right)_{1}=\sup _{\substack{g \in W_{\infty}^{m+1} \\ g\left(t_{1}\right)=\cdots=g\left(t_{2 n}\right)=0}} E\left(g^{(m-r+1)}\right)_{\infty} \leqslant \sup _{\substack{g \in W_{\infty}^{m+1} \\ g\left(t_{1}\right)=\cdots=g\left(t_{2 n}\right)=0}}\left\|g^{(m-r+1)}\right\|_{\infty} .
$$

Применяя теорему 4 при $m=r$, получим

$$
E\left(W_{1}^{r}, S_{2 n, r}^{1}(h)\right)_{1} \leqslant\left\|\varphi_{n, r}\right\|_{\infty},
$$

что в сравнении с соотношением (2.2) при $p=1$ дает равенство (3.2) при $p=1$ и $m=r$.

Для любой функции $g \in W_{\infty}^{m+1}, m \geqslant r+1$, такой, что $g\left(t_{1}\right)=\cdots=g\left(t_{2 n}\right)=0$, имеет место неравенство

$$
\int_{0}^{t} r\left(\left(g^{(m-r+1)}\right)_{ \pm}, u\right) d u \leqslant \int_{0}^{t} r\left(\left(\varphi_{n, r}\right)_{ \pm}, u\right) d u, \quad 0 \leqslant t \leqslant 2 \pi .
$$

Действительно, используя утверждение b) теоремы 4 при $l=m+1$, получим

$$
\left\|g^{(m-r)}\right\|_{\infty} \leqslant\left\|\varphi_{n, r+1}\right\|_{\infty}
$$

Таким образом, выполняются условия теоремы 3.3.4 из [1], в силу которой (4.7) имеет место.

Из (4.7) следует, что функции $g^{(m-r+1)}, m \geqslant r+1$, и $\varphi_{n, r}$ удовлетворяют условиям теоремы 1.3 .13 из [26], из которой следует, что для всех $\lambda \in \mathbb{R}$ и $t \in[0,2 \pi]$ выполняются неравенства

$$
\int_{0}^{t} r\left(\left(g^{(m-r+1)}-\lambda\right)_{ \pm}, u\right), d u \leqslant \int_{0}^{t} r\left(\left(\varphi_{n, r}-\lambda\right)_{ \pm}, u\right), d u .
$$

Теперь, используя предложение 1.3 .14 из [26], можем написать

$$
\int_{0}^{2 \pi} \Pi(f, t) \Pi\left(g^{(m-r+1)}, t\right) d t \leqslant \int_{0}^{2 \pi} \Pi(f, t) \Pi\left(\varphi_{n, r}, t\right) d t .
$$

Из (4.6) и последнего неравенства сразу получаем

$$
E \leqslant \sup _{\substack{f \in F \\ f \perp 1}} \int_{0}^{2 \pi} \Pi(f, t) \Pi\left(\varphi_{n, r}, t\right) d t
$$


О ТОЧНЫХ ЗНАЧЕНИЯХ ПРИБЛИЖЕНИЙ КЛАССОВ ФУНКЦИЙ СПЛАЙНАМИ

Совпадающая с полученной оценкой сверху для $E$ оценка снизу очевидно следует из неравенства

$$
d_{2 n}\left(W^{r} F, L_{1}\right) \leqslant E\left(W^{r} F, S_{2 n, m}\left(\Delta_{h}\right)\right)_{1}
$$

и соотношения (2.4).

Таким образом, соотношение (3.1) доказано. Что касается соотношения (3.2), то оно следует из равенства

$$
\sup _{\substack{\|f\|_{p} \leqslant 1 \\ f \perp 1}} \int_{0}^{2 \pi} \Pi(f, t) \Pi\left(\varphi_{n, r}, t\right) d t=\left\|\varphi_{n, r}\right\|_{p^{\prime}}
$$

Теорема доказана.

ДОКАЗАТЕЛЬСТвО ТЕОРЕМЫ 2. Используя теорему двойственности для наилучшего $L_{1}$-приближения подпространством [1; теорема 1.4.1], получим

$$
E:=E\left(W^{r} H^{\omega}, S_{2 n, m}^{1}(h)\right)_{1}=\sup _{f \in W^{r} H^{\omega}} \sup _{\substack{\|g\|_{\infty} \leqslant 1 \\ g \perp S_{2 n, m}^{1}(h)}} \int_{0}^{2 \pi} f(t) g(t) d t .
$$

Аналогично (4.5) можем написать

$$
E=\sup _{\substack{f \in H^{\omega} \\ f \perp 1}} \sup _{\substack{g \in W_{\infty}^{r} \\ g^{(r)} \perp S_{2 n, m}^{1}(h)}} \int_{0}^{2 \pi} f(t) g(t) d t=\sup _{\substack{f \in H^{\omega} \\ f \perp 1}} \sup _{\substack{g \in W_{\infty}^{m+1} \\ g\left(t_{1}\right)=\cdots=g\left(t_{2 n}\right)}} \int_{0}^{2 \pi} f(t) g^{(m-r+1)}(t) d t .
$$

Через $R(f, t)$ будем обозначать $\Sigma$-перестановку сужения $2 \pi$-периодической функции $f$ на промежуток длины $2 \pi$ [1; с. 294].

Из (4.9), применяя теорему 7.5.1 из [2], получим

$$
E \leqslant \sup _{\substack{g \in W_{\infty}^{m+1} \\ g\left(t_{1}\right)=\cdots=g\left(t_{2 n}\right)=0}} \int_{0}^{2 \pi} R\left(g^{(m-r)}, t\right) \omega^{\prime}(t) d t .
$$

Оценим правую часть (4.10). Пусть сначала $m \geqslant r+2, g \in W_{\infty}^{m+1}$ и

$$
g\left(t_{1}\right)=g\left(t_{2}\right)=\cdots=g\left(t_{2 n}\right)=0 .
$$

Тогда функция $g$ удовлетворяет условиям теоремы 3 , в силу которой

$$
\begin{gathered}
\left\|g^{(m-r)}\right\|_{\infty} \leqslant\left\|\varphi_{n, r+1}\right\|_{\infty}, \\
\max _{a, b \in[0,2 \pi]}\left|\int_{a}^{b} g^{(m-r)}(t) d t\right| \leqslant 2\left\|g^{(m-r-1)}\right\|_{\infty} \leqslant 2\left\|\varphi_{n, r+2}\right\|_{\infty} .
\end{gathered}
$$

Значит, для такой функции $g$ выполняются условия теоремы 6.8.5 из [2] (см. также теорему 7.1.11 из [1]), в силу которой

$$
\int_{0}^{2 \pi} R\left(g^{(m-r)}, t\right) \omega^{\prime}(t) d t \leqslant \int_{0}^{2 \pi} R\left(\varphi_{n, r+1}, t\right) \omega^{\prime}(t) d t .
$$


Пусть теперь $m=r+1$. В этом случае неравенство (4.12) мы установим непосредственно.

Так как $g \in W_{\infty}^{r+2}$ и удовлетворяет условию (4.11), то по теореме 4

$$
\left\|g^{\prime}\right\|_{\infty} \leqslant\left\|\varphi_{n, r+1}\right\|_{\infty}
$$

Теперь в силу теоремы 6.8.4 из [2] заключаем, что для любого $x \in(0, \pi / n)$ выполняется, по крайней мере, одно из неравенств

$$
\left|R^{\prime}\left(g^{\prime}, x\right)\right| \leqslant\left|R^{\prime}\left(\varphi_{n, r+1}, x\right)\right|
$$

или

$$
R\left(g^{\prime}, x\right) \leqslant R\left(\varphi_{n, r+1}, x\right) .
$$

Это означает, что разность $\delta(x)=R\left(\varphi_{n, r+1}, x\right)-R\left(g^{\prime}, x\right)$ на промежутке $[0,2 \pi]$ либо неотрицательна, либо меняет знак с "+" на "-" в единственной точке $t_{0} \in(0,2 \pi)$. Отметим, что в случае, когда $\delta(x) \geqslant 0$ для всех $x \in[0,2 \pi]$, выполнение неравенства (4.12) очевидно. Рассмотрим случай, когда $\delta(x)$ меняет знак на $[0,2 \pi]$.

Обозначим через $g_{j}^{\prime}$ и $\widetilde{\varphi}_{j}^{\prime}$ сужения на $\left[t_{j}, t_{j+2}\right], j=0,2, \ldots, 2 n-2$, функций $g^{\prime}$ и $\widetilde{\varphi}^{\prime}$ соответственно.

В первую очередь отметим, что для всех $j=0,2, \ldots, 2 n-2$ имеет место соотношение

$$
\int_{t_{j}}^{t_{j+1}}\left(g_{j}^{\prime}\right)_{ \pm}(t) d t \leqslant \int_{t_{j}}^{t_{j+1}}\left(\widetilde{\varphi}_{j}^{\prime}\right)_{ \pm}(t) d t .
$$

Действительно, в силу условия $\int_{t_{j}}^{t_{j+1}} g_{j}^{\prime}(t) d t=0$ видим, что $\operatorname{supp}\left(\left(g_{j}^{\prime}\right)_{ \pm}\right) \neq \varnothing$, если $g_{j}^{\prime}$ не тождественный нуль на $\left[t_{j}, t_{j+2}\right]$. Пусть, для определенности,

$$
\operatorname{mes} \operatorname{supp}\left(\left(g_{j}^{\prime}\right)_{+}\right) \geqslant \operatorname{mes} \operatorname{supp}\left(\left(\widetilde{\varphi}_{j}^{\prime}\right)_{ \pm}\right), \quad \operatorname{mes} \operatorname{supp}\left(\left(g_{j}^{\prime}\right)_{-}\right) \leqslant \operatorname{mes} \operatorname{supp}\left(\left(\widetilde{\varphi}_{j}^{\prime}\right)_{ \pm}\right) .
$$

Используя предложение 3.3 .3 из [1] и рассуждения, аналогичные использованным при доказательстве неравенства (4.4), нетрудно убедиться в том, что

$$
\int_{t_{j}}^{t_{j+2}}\left(g_{j}^{\prime}\right)_{-}(t) d t \leqslant \int_{t_{j}}^{t_{j+2}}\left(\widetilde{\varphi}_{j}^{\prime}\right)_{ \pm}(t) d t .
$$

Учитывая (4.16) и тот факт, что

$$
\int_{t_{j}}^{t_{j+2}}\left(g_{j}^{\prime}\right)_{+}(t) d t=\int_{t_{j}}^{t_{j+2}}\left(g_{j}^{\prime}\right)_{-}(t) d t
$$

получим (4.15) и, следовательно,

$$
\int_{0}^{2 \pi}\left|g^{\prime}(t)\right| d t=\int_{0}^{2 \pi}\left|\widetilde{\varphi}^{\prime}(t)\right| d t .
$$

Учитывая тот факт [2; с. 131, 141], что для $\psi \in L_{1}$

$$
\|\psi\|_{1}=\int_{0}^{2 \pi} r(\psi, u) d u=\int_{0}^{2 \pi} R(\psi, u) d u
$$


на основании (4.17) можем утверждать, что $\int_{0}^{2 \pi} \delta(t) d t \geqslant 0$. Пусть $t_{0} \in(0,2 \pi)-$ единственная точка, в которой разность $\delta(x)$ меняет знак с “+" на "-". Рассуждая, как при доказательстве теоремы 6.7.6 из [2], имеем (ниже через $\omega_{+}^{\prime}(t)$ обозначена правая производная функции $\omega(t)$, которая в силу выпуклости вверх функции $\omega(t)$ существует в каждой точке)

$$
\begin{aligned}
\int_{0}^{2 \pi} & \omega^{\prime}(t) R\left(\varphi_{n, r+1}, t\right) d t-\int_{0}^{2 \pi} \omega^{\prime}(t) R\left(g^{\prime}, t\right) d t \\
& =\int_{0}^{2 \pi} \omega^{\prime}(t) \delta(t) d t=\int_{0}^{t_{0}} \omega^{\prime}(t) \delta(t) d t+\int_{t_{0}}^{2 \pi} \omega^{\prime}(t) \delta(t) d t \\
& \geqslant \omega_{+}^{\prime}\left(t_{0}\right) \int_{0}^{t_{0}} \delta(t) d t+\omega_{+}^{\prime}\left(t_{0}\right) \int_{t_{0}}^{2 \pi} \delta(t) d t=\omega_{+}^{\prime}\left(t_{0}\right) \int_{0}^{2 \pi} \delta(t) d t \geqslant 0 .
\end{aligned}
$$

Справедливость (4.12) установлена и при $m=r+1$. Учитывая (4.10), (4.12), а также равенство [1; с. 299]

$$
\left\|f_{n, m}(\omega, \cdot)\right\|_{1}=\int_{0}^{2 \pi} R\left(\varphi_{n, m+1}, t\right) \omega^{\prime}(t) d t
$$

получаем оценку сверху для величин $E\left(W^{r} H^{\omega}, S_{2 n, m}^{1}(h)\right)_{1}$ :

$$
E\left(W^{r} H^{\omega}, S_{2 n, m}^{1}(h)\right)_{1}=E \leqslant \int_{0}^{2 \pi} R\left(\varphi_{n, r+1}, t\right) \omega^{\prime}(t) d t=\left\|f_{n, r}(\omega, \cdot)\right\|_{1} .
$$

Оценка снизу следует из неравенства

$$
E\left(W^{r} H^{\omega}, S_{2 n, r}^{1}(h)\right)_{1} \geqslant d_{2 n}\left(W^{r} H^{\omega}, L_{1}\right)
$$

и $(2.2)$.

Теорема доказана.

ДоКАЗАТЕЛЬСТво тЕОРЕмЫ 3. Сопоставляя (3.2) при $p=1$ и теорему А, мы получаем неравенства (3.4) и (3.5). Из (3.4), (3.5) и $(2.14),(2.15)$ при $r=1,3, \ldots$ и $\delta \geqslant \pi n^{-1}$ получаем соотношения

$$
\kappa_{2 n, r}(\delta)_{1,1}=\kappa_{r}\left(S_{2 n, m}^{1}(h), \delta\right)_{1,1}=\frac{\left\|\varphi_{1, r}\right\|_{\infty}}{2 n^{r}},
$$

а при $r=1,2, \ldots$ и $\delta \geqslant 2 \pi n^{-1}$ соотношения

$$
\kappa_{2 n, r}(\delta)_{1, \infty}=\kappa_{r}\left(S_{2 n, m}^{1}(h), \delta\right)_{1, \infty}=\frac{2\left\|\varphi_{1, r+1}\right\|_{\infty}}{n^{r}} .
$$

Теорема доказана.

В заключение отметим, что результаты работы [8] по приближению классов $W^{r} F$ и классов $W^{r} H^{\omega}$ сплайнами из $S_{2 n, m}^{2}$ в метрике пространства $L_{1}$ можно получить как следствия из теорем 1 и 2 данной статьи с помощью предельного перехода при $h \rightarrow 0$ в соотношениях (3.1) и (3.3). Дело в том, что если для $h \in(0, \pi / n)$ через $s_{h}$ обозначен сплайн из $S_{2 n, m}^{1}(h)$ наилучшего $L_{1}$-приближения функции $f \in L_{1}$, то из семейства $\left\{s_{h}\right\}_{h \in(0, \pi / n)}$ можно выделить последовательность $\left\{s_{h_{k}}\right\}_{k=1}^{\infty}, h_{k} \rightarrow 0$ при 
$k \rightarrow \infty$, которая будет равномерно сходиться к сплайну $s_{0} \in S_{2 n, m}^{2}$. Для этого предельного сплайна будет иметь место соотношение

$$
\left\|f-s_{0}\right\|_{1}=\lim _{k \rightarrow \infty} E\left(f, S_{2 n, m}^{1}\left(h_{k}\right)\right)_{1},
$$

а отсюда уже будет следовать вышесказанное. Однако, реализация этой идеи требует некоторых дополнительных рассмотрений, на которых мы не будем останавливаться в данной работе.

\section{СПИСОК ЦИТИРОВАННОЙ ЛИТЕРАТУРЫ}

[1] Н. П. Корнейчук, Точные константы в теории приближения, Наука, М., 1987.

[2] Н. П. Корнейчук, Экстремалъные задачи теории приближения, Наука, М., 1976.

[3] Н. П. Корнейчук, А. А. Лигун, В. Г. Доронин, Аппроксимация с ограничениями, Наукова думка, Киев, 1982.

[4] М.А. Красносельский, Я. Б. Рутицкий, Выпуклые функции и пространства Орлича, Физматгиз, М., 1958.

[5] С. Г. Крейн, Ю.И. Петунин, Е. М. Семенов, Интерполяиия линейных операторов, Наука, М., 1978.

[6] Х. Трибель, Теория интерполяиии, функииональные пространства, дифференциальные операторы, Мир, М., 1980.

[7] V.F. Babenko, "Approximations, widths and optimal quadrature formulae for classes of periodic functions with rearrangement invariant sets of derivatives", Anal. Math., 13:4 (1987), 281-306.

[8] В. Ф. Бабенко, Н. В. Парфинович, "Точные значения наилучших приближений классов периодических функций сплайнами дефекта 2", Матем. заметки, 85:4 (2009), $538-551$.

[9] С.М. Никольский, "Приближение функций тригонометрическими полиномами в среднем", Изв. АН СССР. Сер. матем., 10:3 (1946), 207-256.

[10] Л. В. Тайков, "О приближении в среднем некоторых классов аналитических функций”, Приближение функций в среднем, Сборник работ, Тр. МИАН СССР, 88, Наука, M., 1967, 61-70.

[11] С. П. Туровец, "О наилучшем приближении в среднем дифференцируемых функций”, Докл. АН УССР. Сер. А, 1968, № 5, 417-421.

[12] A. A. Ligun, "Inequalities for upper bounds of functions", Anal. Math., 2:1 (1976), 11-40.

[13] Ю.И. Маковоз, "Поперечники некоторых функциональных классов в пространстве L", Изв. АН БССР. Сер. физ.-матем. наук, 1969, № 4, 19-28.

[14] Ю. Н. Субботин, "Поперечник класса $W^{r} L$ в $L(0,2 \pi)$ и приближение сплайн-функциями", Матем. заметки, 7:1 (1970), 43-52.

[15] Ю.Н. Субботин, "Приближение сплайн-функциями и оценки поперечников", Приближение периодических функиий, Сборник работ, Тр. МИАН, 109, Наука, М., 1971, $35-60$.

[16] Ю. И. Маковоз, "Об одном приеме оценки снизу поперечников множеств в банаховых пространствах", Матем. сб., 87:1 (1972), 136-142.

[17] А. А. Лигун, "О поперечниках некоторых классов дифференцируемых периодических функций", Матем. заметки, 27:1 (1980), 61-75.

[18] Ю. И. Маковоз, "Поперечники соболевских классов и сплайны, наименее уклоняющиеся от нуля", Матем. заметки, 26:5 (1979), 805-812.

[19] A. Pinkus, "On $n$-widths of periodic functions", J. Analyse Math., 35 (1979), 209-235.

[20] Н. П. Корнейчук, "Верхние грани наилучших приближений на классах дифференцируемых периодических функций в метриках $C$ и $L "$ ", Докл. $A H C C C P$, 190:2 (1970), 269-271. 
[21] Н. П. Корнейчук, "Экстремальные значения функционалов и наилучшее приближение на классах периодических функций”, Изв. АН СССР. Сер. матем., 35:1 (1971), 93-124.

[22] Н.П. Корнейчук, "Наилучшее приближение сплайнами на классах периодических функций в метрике $L "$, Матем. заметки, 20:5 (1976), 655-664.

[23] В. П. Моторный, В. И. Рубан, "Поперечники некоторых классов дифференцируемых периодических функций в пространстве L", Матем. заметки, 17:4 (1975), 531-543.

[24] В.И. Рубан, "Поперечники множеств в пространствах периодических функций", Докл. АН СССР, 225:1 (1980), 34-35.

[25] А. А. Лигун, В. Е. Капустян, Ю. И. Волков, Специалъные вопросы теории приближений и оптимального управления распределенными системами, Выща школа, Киев, 1990.

[26] Н. П. Корнейчук, В. Ф. Бабенко, А. А. Лигун, Экстремалънъе свойства полиномов и сплайнов, Наукова думка, Киев, 1992.

\section{В. Ф. Бабенко}

Днепропетровский национальный университет,

г. Днепропетровск,

Институт прикладной математики и механики НАН Украины,

г. Донецк

E-mail: babenko.vladislav@gmail.com

\section{Н. В. Парфинович}

Днепропетровский национальный университет,

г. Днепропетровск

E-mail: nparfinovich@mail.ru 